АКТГ-ЭКТОПИРОВАННЫЙ СИНДРОМ У ПАЦИЕНТА БЕЗ ВЕРИФИЦИРОВАННОЙ опухоли

(c) Е.В. Ершова, Е.С. Сенюшкина*, Е.А. Трошина

ФГБУ «Национальный медицинский исследовательский центр эндокринологии» Минздрава России, Москва, Россия

Распространенность эндогенного гиперкортицизма составляет около 5-6 случаев на 1 млн человек, доля АКТГ-зависимого гиперкортицизма - порядка 80\%. Среди этой когорты больных у 70-75\% причиной гиперсекреции АКТГ служит кортикотропинома, у 10\% диагностируют гиперплазию кортикотрофов, у оставшихся 15-20\% - нейроэндокринные опухоли различной локализации, продуцирующие АКТГ и/или кортиколиберин. При АКТГ-эктопированном синдроме развитие клинических проявлений, как правило, происходит быстрее, чем при кортикотропиноме, что и определяет своеобразие клинической картины. Трудности выявления первичного очага гиперсекреции АКТГ могут быть обусловлены его малым размером, отсутствием или неспецифичностью клинической картины со стороны первичного очага, ранним метастазированием в случае злокачественных опухолей. Кроме того, клинические проявления гиперкортицизма могут рассматриваться и как самостоятельные нозологические формы, что создает определенные сложности на этапах ранней диагностики и своевременного лечения АКТГ-эктопированного синдрома.

КЛЮЧЕВЫЕ СЛОВА: гиперкортицизм, АКТГ-эктопированный синдром, ожирение, клинический случай.

\title{
SYNDROME OF ECTOPIC ACTH SECRETION IN A PATIENT WITHOUT A VERIFIED TUMOR
}

○ Ekaterina V. Ershova, Evgeniya S. Senyushkina*, Ekaterina A. Troshina

Endocrinology Research Centre, Moscow, Russia

Prevalence of endogenous hypercortisilism is about 5-6 cases per 1 million people. ACTH-dependent hypercortico- sis in the general structure is about $80 \%$. Among this cohort of patients in $70-75 \%$ the cause of hypersecretion of ACTH is corticotrophinoma, about $10 \%$ are diagnosed with hyperplasia of corticotrophs, in the remaining $15-20 \%$ - neuroendocrine tumors of different locations, producing ACTH and / or corticoliberin. With ectopic ACTH syndrome the development of clinical manifestations is faster than with corticotropinoma, which determines the nature of the clinical picture. Difficulties in identifying the primary focus hypersecretion of ACTH may be due to its small size, absence or nonspecificity of the clinical picture from the primary focus, early metastasis in the case of malignant tumors. Besides, clinical manifestations of hyper- cortisolism can be considered and as independent nosological units, which creates certain difficulties at the stages of early diagnosis and timely treatment of ectopic ACTH syndrome.

KEYWORDS: hypercortisolism, ectopic ACTH syndrome, obesity, case report.

\section{АКТУАЛЬНОСТЬ}

Распространенность эндогенного гиперкортицизма составляет около 5-6 случаев на 1 млн человек, при этом ежегодно выявляют 1-2 новых на 1 млн [1]. Доля АКТГ-зависимого гиперкортицизма в общей структуре составляет порядка 80\%. Среди этой когорты больных у 70-75\% причиной гиперсекреции адренокортикотропного гормона (АКТГ) служит кортикотропинома, еще примерно у $10 \%$ диагностируют гиперплазию кортикотрофов, у оставшихся 15-20\% - нейроэндокринные опухоли различной локализации, продуцирующие АКТГ и/или кортиколиберин [1-4].

Впервые больная с овсяноклеточным раком легких и клинической картиной гиперкортицизма была описана в 1928 г. W. Brown [5]. В 1960 г. выделены пептиды из опухоли легкого, обладающие адренокортикотропной активностью, а в 1969 г. G. Liddle и соавт. доказали патогенетическую связь между гиперкортицизмом и функциональной активностью некоторых типов опухолей, что позволило выделить новое заболевание под названием «АКТГ-эктопированный синдром» [6].
Согласно эпидемиологическим данным, в 5-10\% случаев источником эктопической продукции АКТГ являются опухоли внегипофизарной локализации: карциноидные опухоли бронхов (36-46\%), овсяноклеточный рак легких (18-20\%), феохромоцитома (9-23\%), медуллярный рак щитовидной железы (3-7\%), опухоли поджелудочной железы, вилочковой и околоушной железы, яичников, матки, предстательной железы, толстой кишки, желудка, пищевода и других органов [3, 7]. При АКТГ-эктопированном синдроме развитие клинических проявлений, как правило, происходит быстрее, чем при кортикотропиноме, что определяет своеобразие клинической картины [4]. Трудности выявления первичного очага гиперсекреции АКТГ могут быть обусловлены его малым размером, отсутствием или неспецифичностью клинической картины со стороны первичного очага, ранним метастазированием в случае злокачественных опухолей. Кроме того, наиболее часто встречающиеся проявления гиперкортицизма (такие как артериальная гипертензия, миопатия, нарушения углеводного обмена, в т.ч. сахарный диабет (СД), электролитные расстройства и усиленный катаболизм белков, способствующие 
развитию миопатии и низкотравматичным переломам, гипогонадизм и т.д.) могут рассматриваться иногда как самостоятельные нозологические формы, что создает определенные сложности на этапах ранней диагностики и своевременного лечения АКТГ-эктопированного синдрома. Приведенный клинический случай - тому подтверждение.

\section{ОПИСАНИЕ СЛУЧАЯ}

Пациент П., 36 лет, в ноябре 2016 г. поступил в ФГБУ ЭНЦ с жалобами на повышение артериального давления (АД) максимально до 210/140 мм рт.ст., боль в грудной клетке и межреберьях при физической нагрузке, отеки и онемение рук и ног, прибавку в весе, бордовые стрии в подмышечных впадинах, на животе и бедрах, «синяки» на коже, длительное заживление ран, изменение внешности (округление и покраснение лица), мышечную слабость, одышку при физической нагрузке, усталость, слабость, рассеянность, головную боль.

Из анамнеза известно, что в мае 2015 г. в возрасте 35 лет после незначительной физической нагрузки возник компрессионный перелом в грудном отделе позвоночника на уровне Th5-Th6 (по данным МРT), патологические переломы ребер с обеих сторон. В июне 2015 г. при проведении денситометрии проксимального отдела бедра Z-критерий -0,3 SD, поясничного отдела позвоночника Z-критерий -1,3 SD. В октябре 2015 г. при обследовании выявлены: СД, гликемия в дебюте 17 ммоль/л без какой-либо клинической симптоматики, расценен как СД 1 типа, назначена инсулинотерапия; отрицательный ночной подавляющий тест (НПТ) с 1 мг дексаметазона (кортизол крови 556 нмоль/л) при нормальном суточном анализе мочи на свободный кортизол - 157,2 мкг/сут (норма 4,3-176) и отсутствии органических изменений надпочечников при КТ с внутривенным контрастированием; уровень метилированных катехоламинов в суточной моче в норме. В марте 2015 г. определен низкий уровень тестостерона. Однако несмотря на развернутую клиническую картину гиперкортицизма (стрии, «синяки» на коже, округление и покраснение лица, длительное заживление ран, мышечная слабость, переломы позвонков и ребер, СД, гипогонадизм) и отсутствие подавления кортизола на фоне НПТ с 1 мг дексаметазона, диагностических и лечебных мероприятий не проводилось на протяжении года (до апреля 2016 г.).

В апреле 2016 г.: кортизол крови - 1511,8 нмоль/л (норма 260-720), АКТГ - 38,4 мкг/л (норма 0-50), 25(ОН)D -

Таблица 1. Гормональные анализы в развернутую стадию заболевания

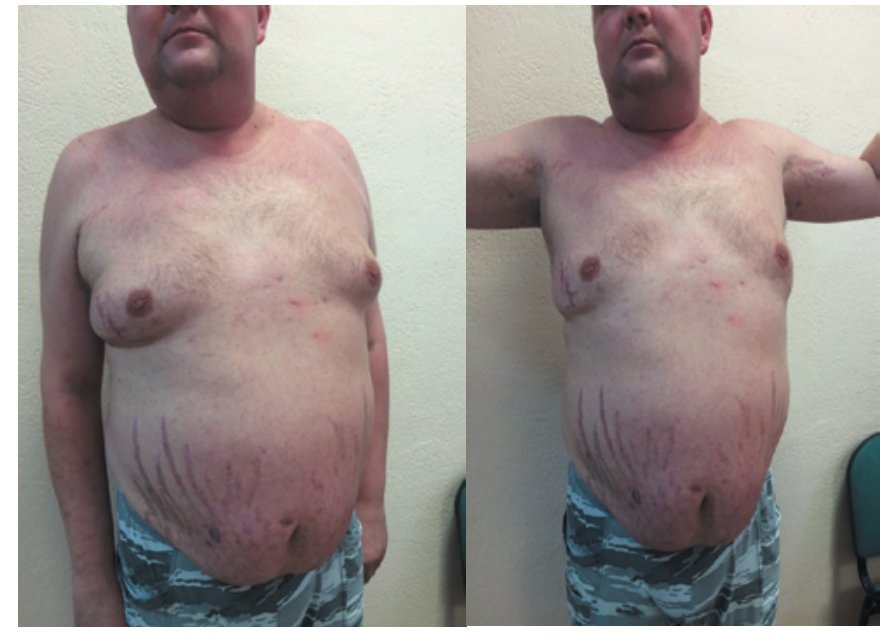

Рис. 1. Внешний вид больного П. на момент поступления в ФГБУ ЭНЦ (ноябрь 2016 г.)

12,5 нг/мл (норма более 30); на МРТ гипофиза: патологических изменений гипофиза не выявлено.

Результаты физикального, лабораторного

и инструментального исследования

При поступлении в ФГБУ ЭНЦ (ноябрь 2016 г.): рост 184 см, вес 115 кг, ИМТ 34 кг/м². Кожные покровы: матронизм, багровые стрии в подмышечных впадинах, на животе и бедрах, отеки голеней (рис. 1).

С целью подтверждения гиперкортицизма и дифференциальной диагностики в рамках синдрома проведено обследование (табл. 1, 2).

Отрицательный НПТ с 1 мг дексаметазона (выполненный по месту жительства), высокий уровень кортизола в суточной моче и слюне вечером подтверждали наличие у пациента эндогенного гиперкортицизма. Повышенный уровень АКТГ в вечернее время с инвертированным суточным ритмом свидетельствовал об АКТГ-зависимом гиперкортицизме. Отрицательная большая дексаметазоновая проба (БДП) с 8 мг дексаметазона и отсутствие аденомы гипофиза по данным МРТ (нельзя исключить диффузные аденоматозные изменения структуры аденогипофиза) позволили заподозрить АКТГ-эктопированный синдром.

В общем анализе крови отмечался типичный для синдрома гиперкортицизма лейкоцитоз без повышения СОЭ. По данным денситометрии - в поясничном отделе позвоночника (total) Z-критерий -1,3 SD, в проксимальном отделе бедра (total) Z-критерий $-0,7$ SD, в лучевой кости (total) Z-критерий -0,5 SD.

\begin{tabular}{lccc}
\hline \multicolumn{1}{c}{ Показатель } & Значение & $\begin{array}{c}\text { Единицы } \\
\text { измерения }\end{array}$ & $\begin{array}{c}\text { Референсный } \\
\text { интервал }\end{array}$ \\
\hline Кортизол св. (моча)/сут & 3233,6 & нмоль/сут & $60-413$ \\
Кортизол в слюне (вечер) & 100,8 & нмоль/л \\
Кортизол (кровь) до большой дексаметазоновой & 1750 & нмоль/л \\
пробы (БДП) (с 8 мг дексаметазона) & & нололь/л \\
Кортизол (кровь) после БДП (с 8 мг дексаметазона) & 1506 (снижение на 14\%) & но\% при БИК \\
АКТГ (утро) & 61,78 & пг/мл & $7-66$ \\
АКТГ (вечер) & 77,82 & пг/мл & $0-30$ \\
\hline
\end{tabular}


Таблица 2. Биохимические показатели в динамике заболевания

\begin{tabular}{|c|c|c|c|c|}
\hline Показатель & Ноябрь 2016 г. & Июль 2017 г. & $\begin{array}{c}\text { Единицы } \\
\text { измерения }\end{array}$ & $\begin{array}{c}\text { Референсный } \\
\text { интервал }\end{array}$ \\
\hline Глюкоза & 12,71 & 5,21 & ммоль/л & $3,1-6,1$ \\
\hline Мочевая кислота & 421 & 536 & мкмоль/л & $202-416$ \\
\hline Холестерин общ. & 7,18 & 4,02 & ммоль/л & $3,3-5,2$ \\
\hline ЛПНП & 4,2 & 2,08 & ммоль/л & $1,1-3$ \\
\hline Триглицериды & 4,26 & 2,74 & ммоль/л & $0,1-1,7$ \\
\hline Креатинин & 95,8 & 81 & мкмоль/л & $63-110$ \\
\hline Натрий & 146 & 138 & ммоль/л & $136-145$ \\
\hline Калий & 2,5 & 4,4 & ммоль/л & $3,5-5,1$ \\
\hline Мочевина & 7,5 & 7,2 & ммоль/л & $3,2-7,4$ \\
\hline Гликированный гемоглобин & 10,8 & 6,8 & $\%$ & $4,8-6$ \\
\hline Альбумин моча (раз. порция) & 72 & 54 & мг/л & $0-20$ \\
\hline
\end{tabular}

На основании клинической картины и лабораторно-инструментальной диагностики пациенту был выставлен клинический диагноз.

Код МКБ: Е24.3 Эктопический АКТГ- синдром

АКТГ-зависимый гиперкортицизм. АКТГ-эктопированный синдром. Сахарный диабет вследствие АКТГ-зависимого гиперкортицизма. Трофическая язва левой голени. Диабетическая нефропатия. ХБП C2 A2. Стероидная миопатия. Остеопороз тяжелого течения с компрессионными переломами Th5-Th7 и патологическими переломами ребер с двух сторон. Артериальная гипертензия II cm., риск 4. ХСН II, ФК II. Стероидная кардиомиопатия. Гипертоническая ангиопатия сетчатки ОU. Ожирение I cm. Дислипидемия. Неалкогольная жировая болезнь печени. Недостаточность витамина D. Гиперурикемия. Мочекаменная болезнь.

Для уточнения генеза АКТГ-зависимого гиперкортицизма [8] пациенту проведен селективный венозный забор крови из нижних каменистых синусов (табл. 3). Его результаты продемонстрировали отсутствие достаточного градиента АКТГ ( $\geq 2$ до стимуляции и $\geq 3$ после стимуляции) между синусами и периферией (максимальный градиент 2,71 между правым синусом и периферией достигнут на 3-й минуте стимуляции десмопрессином), что было расценено как АКТГ-эктопированный синдром (табл. 3).
В соответствии с результатами селективного забора крови, свидетельствующими об отсутствии центрального генеза АКТГ-зависимого гиперкортицизма, проведен поиск возможного очага АКТГ-эктопии: мультиспиральная компьютерная томография (МСКТ) органов грудной полости, средостения, брюшной полости, забрюшинного пространства и органов малого таза - очаговых изменений не выявлено; УзИ щитовидной железы и брюшной полости - очаговой патологии не выявлено; эзофагогастродуоденоскопия (ЭГДС) - признаки кандидозного эзофагита, эрозивный антральный гастрит; колоноскопия патологии не выявлено.

Завершающим этапом поиска источника АКТГ-эктопии стало проведение позитронно-эмиссионной томографии с ${ }^{18} \mathrm{~F}$-фтордезоксиглюкозой, по данным которой, от верхней части свода черепа до середины бедра очаги

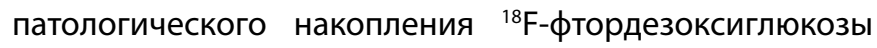
не определяются.

Таким образом, отсутствие четких данных за наличие аденомы гипофиза, а также отсутствие визуализации возможного источника АКТГ-эктопии при проведенном топическом поиске явились основаниями для проведения пациенту по жизненным показаниям двусторонней адреналэктомии.

В январе 2017 г. в хирургическом отделении ФГБУ ЭНЦ пациенту проведена лапароскопическая двусторонняя адреналэктомия. Морфологическое исследование по-

Таблица 3. Селективный венозный забор крови из нижних каменистых синусов

\begin{tabular}{lccccc}
\hline Время & $\begin{array}{c}\text { Правый } \\
\text { синус }\end{array}$ & $\begin{array}{c}\text { Левый } \\
\text { синус }\end{array}$ & Периферия & $\begin{array}{c}\text { Максимальный градиент } \\
\text { между центром и периферией } \\
\text { до и после стимуляции }\end{array}$ & $\begin{array}{c}\text { Максимальный градиент между } \\
\text { правым и левым синусами } \\
\text { до и после стимуляции }\end{array}$ \\
\hline & & & АКТГ & 1,14 \\
-5 мин & 58,4 & 51,2 & 46,8 & 1,24 & 1,14 \\
О мин & 52,8 & 46,3 & 41,0 & 1,28 & 1,17 \\
+3 мин & 219,2 & 186,6 & 80,6 & 2,71 & 1,00 \\
+5 мин & 235,5 & 236,6 & 152,4 & 1,55 & 1,01
\end{tabular}

Пролактин

$\begin{array}{llll}-5 & \text { мин } \quad 1150,0 \quad 427,3 \quad 254,1\end{array}$

4,52

2,69 
слеоперационного материала: диффузно-микронодулярная гиперплазия коры надпочечников, в основном за счет пучковой зоны, представленная гипертрофированными клетками с компактной эозинофильной цитоплазмой, в некоторых из них гранулы липофусцина, отмечается гиперплазия мозгового слоя надпочечника. При проведении иммуногистохимического исследования в мозговом слое надпочечника отдельные клетки и группы клеток экспрессируют АКТГ (около 30\% клеток мозгового слоя); отмечается слабая экспрессия АКТГ В коре надпочечника, преимущественно в перимедуллярном пучковом слое коры.

В послеоперационном периоде назначены глюкои минералокортикоиды (гидрокортизон 20 мг утром, 15 мг днем, 10 мг вечером; флудрокортизон 0,05 мг утром). На этом фоне проявлений надпочечниковой недостаточности не было.

Через 4 месяца после проведенной двусторонней адреналэктомии (рис. 2) у пациента отмечено снижение массы тела на 15 кг, побледнение стрий, исчезновение гиперемии и отечности лица и «синяков» на коже, существенное уменьшение мышечной слабости, постепенная нормализация АД до 110-120/70-80 мм рт.ст., что позволило откорректировать гипотензивную терапию; наблюдалась тенденция к снижению гликемии натощак и постпрандиально до субнормальных показателей и снижение уровня гликированного гемоглобина (от 10,8 до 6,8\%), в связи с чем отменен инсулин пролонгированного действия и уменьшены дозы инсулина короткого действия; на фоне приема 20 мг аторвастатина выявлена значимая положительная динамика в отношении показателей липидного спектра крови (общий холестерин снизился от 7,18 до 4,02 ммоль/л, ЛПНП от 4,2 до 2,08 ммоль/л, триглицериды от 4,26 до 2,74 ммоль/л) и нормокалиемия (табл. 2). Данные изменения позволили уменьшить дозу гидрокортизона. Отсутствие положительных сдвигов в отношении гиперурикемии свидетельствует об отсутствии нормализации пуринового обмена и диктует необходимость продолжить гипопуриновую диету на фоне расширения питьевого режима. Сохранялись повышенные показатели АКТГ (утро 91,26 пг/мл, вечер 147,5 пг/мл) на фоне приема 45 мг гидрокортизона.

\section{ОБСУЖДЕНИЕ}

Данное клиническое наблюдение в первую очередь диктует необходимость обратить внимание врачей на несвоевременную диагностику заболевания у данного пациента даже при развернутой клинической картине гиперкортицизма (бордовые стрии, «синяки» на коже, округление и покраснение лица на фоне прибавки массы тела, длительное заживление ран, мышечная слабость, переломы позвонков, сахарный диабет, низкий уровень тестостерона) и отрицательном НПТ с 1 мг дексаметазона, что отсрочило постановку диагноза на 6-12 мес и могло привести к развитию тяжелых инвалидизирующих осложнений.

Кроме того, представленный случай АКТГ-эктопированного синдрома демонстрирует сложности диагностики данной патологии, которые заключаются не столько в самой постановке диагноза АКТГ-эктопированного синдрома, сколько в поиске очага эктопической секре-

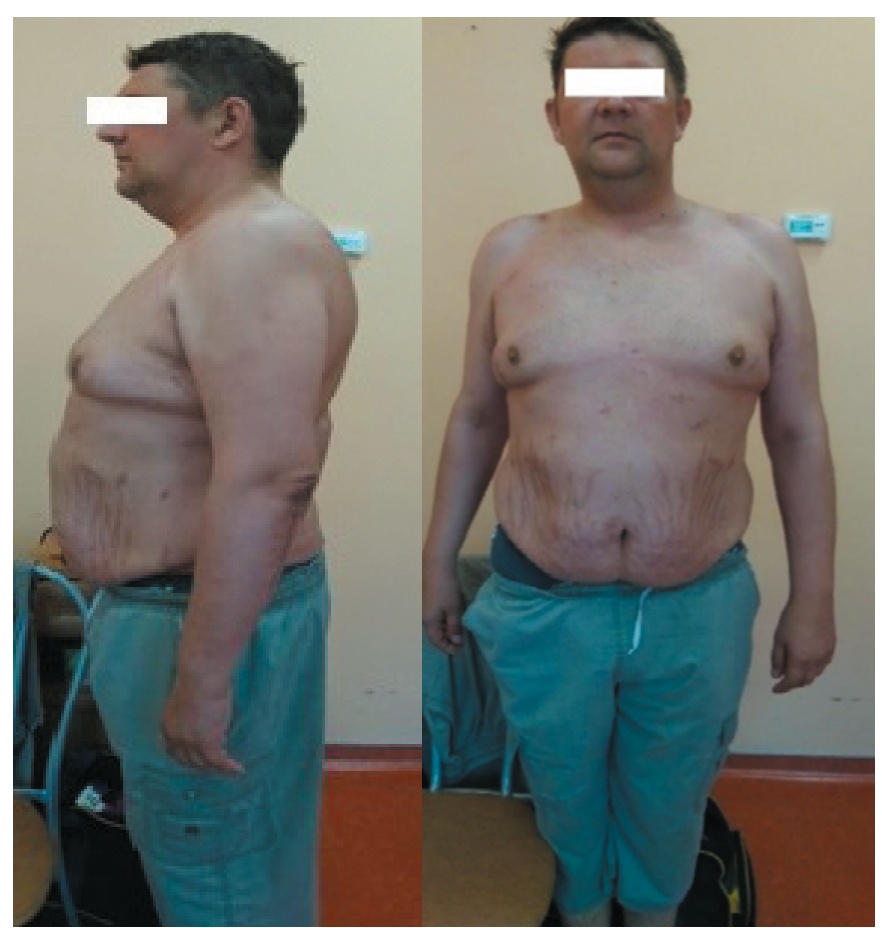

Рис. 2. Внешний вид больного П. через 4 месяца после двусторонней адреналэктомии

ции АКТГ. К сожалению, небольшие размеры первичного очага и отсутствие или неспецифичность клинической картины именно со стороны очага АКТГ-эктопии часто не позволяют выявить опухоль на ранних стадиях, а современные методы топической диагностики не всегда обладают достаточной чувствительностью.

В нашем случае тяжесть состояния пациента, обусловленная наличием развернутой клинической картины гиперкортицизма, при отсутствии источника АКТГ-эктопии, несмотря на тщательный диагностический поиск, в т.ч. проведение позитронно-эмиссионной то-

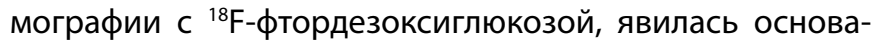
нием для проведения лапароскопической двусторонней адреналэктомии. Согласно литературе, такую лечебную тактику приходится выбирать у 13\% больных с АКТГ-эктопированным синдромом [9-10].

Выявленная в нашем клиническом примере двусторонняя гиперплазия мозгового слоя надпочечников и результаты иммуногистохимического исследования (около 30\% клеток мозгового слоя надпочечников экспрессируют АКТГ) с большой долей вероятности позволяют рассматривать ее в качестве возможного очага АКТГ-эктопии с соответствующей диффузно-микронодулярной гиперплазией коры надпочечников, в основном за счет пучковой зоны. По данным литературы [9, 11, 12], на долю феохромоцитом действительно приходится по меньшей мере 2,3-5,6\% всех случаев АКТГ-эктопий. Однако у данного пациента феохромоцитома была исключена на дооперационном этапе на основании нормального уровня метилированных катехоламинов в суточной моче и отсутствия очагов патологического накопления ${ }^{18} \mathrm{~F}$-фтордезоксиглюкозы при позитронно-эмиссионной томографии. Полученные результаты дают возможность предположить, что гиперплазия мозгового слоя надпочечников была лишь предшественником феохромоцитомы, в нашем случае - двусторонней. В литературе нам удалось найти описание схожего случая АКТГ-эк- 
топии только в одном случае [12]. Выявленная диффузно-микронодулярная гиперплазия коры надпочечников не противоречит результатам морфологического исследования послеоперационного материала, представленным другими авторами [13].

Поскольку одним из лабораторных критериев эффективности лечения АКТГ-эктопированного синдрома является снижение уровня АКТГ, сохраняющиеся у пациента П. повышенные уровни АКТГ в утреннее и вечернее время (на фоне приема 45 мг гидрокортизона), с одной стороны, могут свидетельствовать о сохранении источника АКТГ-эктопии, что требует дальнейшего динамического наблюдения и продолжения топической диагностики для поиска первичного очага, с другой - могут рассматриваться и как вариант нормы при ответе здорового гипофиза на двустороннюю адреналэктомию.

\section{ЗАКЛЮЧЕНИЕ}

Таким образом, диагностика АКТГ-эктопированного синдрома, поиск первичного очага и в настоящее время, несмотря на наличие современных визуализирующих методов диагностики, представляют немалые трудности и зачастую требуют проведения длительного динамического наблюдения и дальнейшего топического поиска уже после двусторонней адреналэктомии.

\section{ДОПОЛНИТЕЛЬНАЯ ИНФОРМАЦИЯ}

Согласие пациента. Пациент добровольно подписал информированное согласие на публикацию персональной медицинской информации в обезличенной форме в журнале «Ожирение и метаболизм».

Конфликт интересов. Авторы заявляют об отсутствии конфликта интересов.

\section{СПИСОК ЛИТЕРАТУРЫ | REFERENCES}

1. Эндокринология: национальное руководство / Под ред. Дедова И.И., Мельниченко Г.А.. - 2-е изд., перераб. и доп. - М.: ГЭОТАР-Медиа, 2016. [Dedov II, Melnochenko GA, editors. Endokrinologiya: nacional'noe rukovodstvo. Moscow: GEHOTAR-Media; 2016. (In Russ.)]

2. Nieman LK, Biller BMK, Findling JW, et al. The Diagnosis of Cushing's Syndrome: An Endocrine Society Clinical Practice Guideline. J. Clin. Endocr. Metab. 2008;93(5):1526-1540. doi: 10.1210/jc.2008-0125.

3. Гуревич Л.Е., Воронкова И.А., Марова Е.И., и др. Клинико-морфологическая характеристика АКТГ-продуцирующих опухолей различной локализации с эктопическим синдромом Кушинга. // Альманах клинической медицины. - 2017. - № 4. - C. 289-301. [Gurevich LE, Voronkova IA, Marova El, et al. Clinical and morphological characteristic of ACTH producing tumors of various localization and the ectopic Cushing's syndrome. Almanac of Clinical Medicine. 2017;45(4):289-301. (in Russ.)] doi: 10.18786/2072-0505-2017-45-4-289-301.

4. Эндокринология. / Под ред. Дедова И.И., Мельниченко Г.А., Фадеева В.В. М.: ГЭОТАР-Медиа; 2009;. [Dedov II, Mel'nichenko GA, Fadeev W, editors. Endokrinologiya. Moscow: GEOTAR-Media; 2009. (In Russ.)]

5. Brown WH. A case of pluriglandular syndrome. "Diabetes of bearded women". Lancet. 1928; 2: 1022-23.

6. Liddle GW, Nicholson WE, Island DP, et al. Clinical and laboratory studies of ectopic humoral syndromes. Recent Prog. Horm. Res. 1969; 25: 283-324.

7. Ветшев П.С., Мельниченко Г.А., Павлова М.Г., и др. Клиническое наблюдение АКТГ-эктопированного синдрома. // Хирургия. 2006. - № 9. - C. 63-65. [Vetshev PS, Mel'nichenko GA, Pavlova MG, et al. Klinicheskoe nablyudenie AKTG-ehktopirovannogo sindroma. Hirurgiya. 2006;(9):63-65. (In Russ.)]

8. Протокол оказания медицинской помощи в рамках клинической апробации: Оказание_медицинской_помощи_пациентам_с_АКТГ-зависимым_эндогенным_гиперкортицизмом [доступ от 01.09.2018] https://www.rosminzdrav.ru/poleznye-resursy/ protokoly-klinicheskoy-aprobatsii/realizuemye-protokoly-klinicheskoy-aprobatsii [интернет]

9. Гуревич Л.Е., Воронкова И.А., Марова Е.И. и соавт. Клинико-морфологическая характеристика АКТГ-продуцирующих опухолей различной локализации с эктопическим синдромом Кушинга // Альманах клинической медицины. - 2017. - Т. 45. - № 4. C. 289-301. [Gurevich LE, Voronkova IA, Marova El, et al. Clinical and morphological characteristic of ACTH producing tumors of various localization and the ectopic Cushing's syndrome. Almanac of Clinical Medicine. 2017:45(4):289-301. (In Russ.)] doi: 10.18786/2072-05052017-45-4-289-301.

10. Российские клинические рекомендации. Эндокринология / Под ред. Дедова И.И., Мельниченко Г.А.. - М.: ГЭОТАР-Медиа; 2016. [Dedov II, Mel'nichenko GA, editors. Rossijskie klinicheskie rekomendatsii. Endokrinologiya. Moscow: GEOTAR-Media; 2016.]

11. Кузнецов Н.С., Марова Е.И., Латкина Н.В. и соавт. АКТГ-продуцирующая феохромоцитома. Клинический случай // Эндокринная хирургия. - 2012. - Т. 6. - №4. - С. 43-50. [Kuznetsov NS, Marova El, Latkina NV, et al. ACTH-Secreting Pheochromocytoma. Case report. Endocrine Surgery. 2012;6(4):43. (In Russ.)] doi: 10.14341/2306-3513-2012-4-43-50.

12. Falhammar H, Calissendorff J, Höybye C. Frequency of Cushing's syndrome due to ACTH-secreting adrenal medullary lesions: a retrospective study over 10 years from a single center. Endocrine. 2016;55(1):296-302. doi: 10.1007/s12020-016-1127-y.

13. Дзеранова Л.К., Михайлова Д.С., Рожинская Л.Я., и др. АКТГ-эктопированный синдром у пациентки с карциноидом легкого // Ожирение и метаболизм. - 2009. - Т. 6. - №3. - C. 54-58. [Dzeranova LK, Mikhailova DS, Rozhinskaya L, et al. ACTH-ectopic syndrome in patient with lung carcinoid. Obesity and metabolism. 2009; (3):54 (In Russ.)] doi: 10.14341/2071-8713-5246.

\section{ИНФОРМАЦИЯ ОБ АВТОРАХ [AUTHORS INFO]}

*Сенюшкина Евгения Семеновна, клинический ординатор [Evgeniya S. Senyushkina, clinical resident]; адрес: Россия, 117036, Москва, ул. Дм. Ульянова, д. 11 [address: 11 Dm. Ulyanova street, 117036 Moscow, Russia]; ORCID: https://orcid.org/0000-0001-7960-8315; eLibrary SPIN: 4250-5123; e-mail: EvgeniyaSenyushkina@yandex.ru

Ершова Екатерина Владимировна, к.м.н., [Ekaterina V. Ershova, MD, PhD]; ORCID: https://orcid.org/0000-0002-6220-4397;; eLibrary SPIN: 6728-3764; e-mail: yu99pol06@rambler.ru Трошина Екатерина Анатольевна, д.м.н., член-корр. PAH, профессор [Ekaterina A. Troshina, MD, PhD, Professor]; ORCID: https://orcid.org/0000-0002-8520-8702; eLibrary SPIN: 8821-8990; e-mail: troshina@inbox.ru 


\section{ЦИТИРОВАТЬ:}

Ершова Е.В., Сенюшкина Е.С., Трошина Е.А. АКТГ-эктопированный синдром у пациента без верифицированной опухоли // Ожирение и метаболизм. — 2018. - Т.15. — №. 3 - С. 59-64. doi: 10.14341/omet9402

\section{TO CITE THIS ARTICLE:}

Ershova E.V, Senyushkina ES, Troshina EA. Syndrome of ectopic ACTH secretion in a patient without a verified tumor. Obesity and metabolism. 2018;15(3):59-64. doi: 10.14341/omet9402 